\title{
Preconditioned Krylov Subspace Methods Solving Dense Nonsymmetric Linear Systems Arising from BEM
}

\author{
Zejun Chen and Hong Xiao \\ College of Mechanical Engineering, Yanshan University, Qinhuangdao 066004, China \\ chen_zejun@163.com, xhh@ysu.edu.cn
}

\begin{abstract}
Discretization of boundary integral equations leads, in general, to fully populated nonsymmetric linear systems of equations. We research the comparative performances of iterative techniques based on Krylov subspace solvers as $\operatorname{GMRES}(m)$, QMR and Bi-CGStab solving linear systems arising from BEM elasticity problems. Several general preconditioners are also considered and assessed. The results of numerical experiments suggest that preconditioned Krylov subspace methods are effective approaches for the solution of dense nonsymmetric linear systems arising from BEM.
\end{abstract}

Keywords: Krylov Subspace Method; Preconditioner; Dense Nonsymmetric Matrix; Boundary Element Method.

\section{Introduction}

The Boundary Element Method (BEM) is an important technique in the numerical solution of engineering or scientific problems. The matrix of the system in the BEM is fully populated and nonsymmetric. A fast convergent iterative solution method, which would require the number of iterations much less than the number of DOF, will decrease the computational cost to $O\left(N^{2}\right)$ [1]. The Krylov subspace iterative methods are efficient methods in solving the linear systems arising from BEM formulation [2-4]. On the other hand, the systems obtained from the discretization step are badly conditioned, especially when mixed boundary conditions exist or large scale problems. Thereby, preconditioning is very essential to the successful use of Krylov subspace iterative methods. In this paper, we are primarily concerned with the efficient solution of dense nonsymmetric systems arising from BEM elasticity problems by preconditioned Krylov subspace methods.

\section{Preconditioned Krylov Subspace Methods}

We are concerned with the solution of dense nonsymmetric linear systems $A x=b$ arising from BEM formulation. In this paper, three of the most widely used Krylov subspace methods (GMRES, QMR, Bi-CGStab) are considered. Briefly, starting with an initial guess $x_{0}$, iterates $x_{i} \in x_{0}+K_{i}\left(A, r_{0}\right)$ are produced such that the residuals $r_{i}=b-A x_{i}$ are in some sense small [4]. The Krylov subspace is defined as 


$$
K_{m}\left(A, r_{0}\right)=\operatorname{span}\left\{r_{0}, A r_{0}, A^{2} r_{0}, \cdots, A^{m-1} r_{0}\right\}
$$

Note that these subspaces are nested, i.e. $K_{m} \subseteq K_{m+1}$.

It is well known that the rate of convergence of an iterative method depends greatly on the spectral properties of coefficient matrix. Hence, iterative methods usually involve a matrix that transforms the original coefficient matrix into one having the same solution but more favorable spectral properties. A preconditioner is a matrix that can be used to accomplish such a transformation.

$$
M^{-1} A x=M^{-1} b
$$

In practice, the preconditioner should meet the requirement of two characteristics. First, the preconditioned matrix $M^{-1} A$ must be (much) better conditioned than $A$ so that Eq.(2) can be efficiently solved by iterative methods. Secondly, the cost of constructing and applying preconditioner must be cheap. However, the two requirements are contradicting, so there is a trade-off between the cost of constructing and applying the preconditioner, and the improvement of iterative efficiency.

\section{Numerical Examples}

The experiments with different krylov subspace methods are performed using the iterative solver written in Fortran90 and compiled by Compaq Visual Fortran 6.5. All experiments are performed on a PC with 1GB RAM memory under Windows XP Professional. The computer is equipped with a $2.8 \mathrm{GHz}$ Intel Pentium 4 processor and no special software optimization or specialized hardware. A convenient stopping criteria is used the stopping criteria $i>$ maxit or $\left\|r^{(i)}\right\| \leq \varepsilon \cdot\|b\|$.

Example 1: In this section, we solve the same numerical problem using different preconditioned Krylov subspace methods. The number of DOF is 1806 . The initial guess is the zero vector. The convergence histories of Krylov subspace methods are depicted in Figs. 1-3 for different preconditioning techniques gives us some clues as to the numerical behaviors of the proposed schemes. In these figures "JOBI" means Jacobi preconditioning, "BJOB" means Block Jacobi preconditioning, "ILU3" and "ILU5" mean Incomplete LU Factorization Preconditioning with Tri-diagonal and Five-diagonal nonzero structure, respectively.

From these figures, it can be seen that the convergence velocity of Krylov subspace methods can be significantly improved by using the preconditioning techniques. The residual of Jacobi preconditioned Krylov subspace methods tends to decrease very rapidly compared with the ILU preconditioning techniques. Among these Krylov subspace methods, the residues of GMRES methods decrease monotonically and rapidly with the increasing iteration number, the convergence behaviors of other methods are quite irregular, and may even break down.

Example 2: In this section, we choose the three methods to calculate different DOFs of BEM problem. In table 1, we describe the results of experiments on an elasticity 


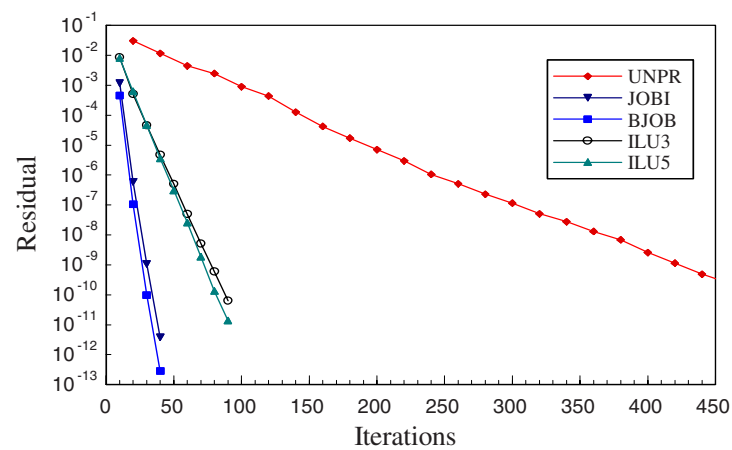

Fig. 1. Convergence histories of GMRES for different preconditioning techniques

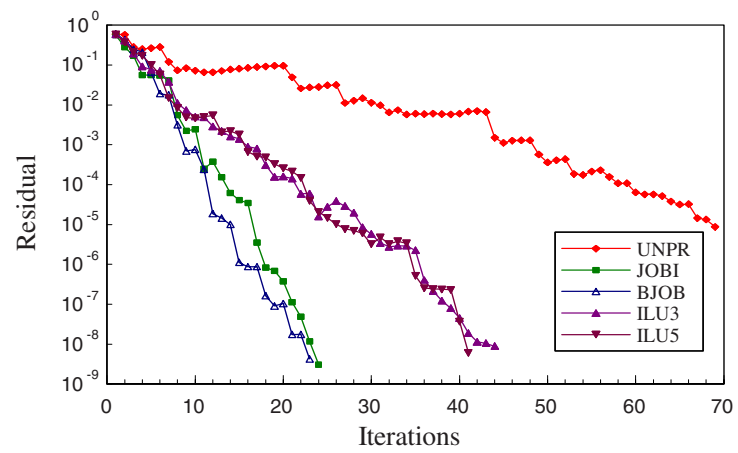

Fig. 2. Convergence histories of QMR for different preconditioning techniques

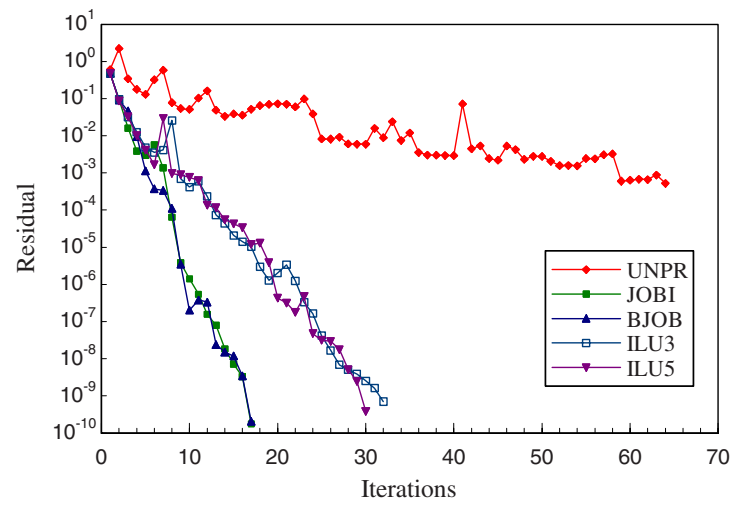

Fig. 3. Convergence histories of Bi-CGStab for different preconditioning techniques

problem with different DOFs for three Krylov subspace methods in conjunct with the Block Jacobi preconditioning scheme. Here, the initial guess is the zero vector, the convergence criterion $\varepsilon=\left\|r^{(i)}\right\| /\|b\| \leq 1.0 \times 10^{-10}$. 
Table 1. The CPU time (in seconds) and iterations of krylov subspace methods

\begin{tabular}{|c|c|c|c|c|c|c|}
\hline \multicolumn{2}{|c|}{ Solvers } & \multicolumn{5}{c|}{ DOF } \\
\cline { 3 - 7 } & \cline { 3 - 7 } & 1,158 & 1,806 & 4,056 & 4,614 & 7,206 \\
\hline \multirow{3}{*}{ GMRES(30) } & Residual & $6.81 \mathrm{E}-12$ & $5.38 \mathrm{E}-12$ & $4.13 \mathrm{E}-11$ & $4.17 \mathrm{E}-11$ & $7.57 \mathrm{E}-11$ \\
\cline { 2 - 7 } & CPU time & $4.78 \mathrm{~s}$ & $10.63 \mathrm{~s}$ & $89.14 \mathrm{~s}$ & $114.25 \mathrm{~s}$ & $293.29 \mathrm{~s}$ \\
\cline { 2 - 7 } & Iterations & 30 & 30 & 30 & 30 & 30 \\
\hline \multirow{3}{*}{ QMR } & Residual & $7.20 \mathrm{E}-11$ & $3.35 \mathrm{E}-11$ & $2.72 \mathrm{E}-11$ & $3.97 \mathrm{E}-11$ & $8.33 \mathrm{E}-11$ \\
\cline { 2 - 7 } & CPU time & $4.85 \mathrm{~s}$ & $11.41 \mathrm{~s}$ & $138.17 \mathrm{~s}$ & $182.40 \mathrm{~s}$ & $514.72 \mathrm{~s}$ \\
\cline { 2 - 7 } & Iterations & 26 & 29 & 31 & 32 & 36 \\
\hline \multirow{3}{*}{ Bi-CGStab } & Residual & $5.85 \mathrm{E}-11$ & $6.52 \mathrm{E}-12$ & $8.82 \mathrm{E}-11$ & $4.62 \mathrm{E}-10$ & $8.79 \mathrm{E}-11$ \\
\cline { 2 - 8 } & CPU time & $4.57 \mathrm{~s}$ & $10.64 \mathrm{~s}$ & $101.51 \mathrm{~s}$ & $126.69 \mathrm{~s}$ & $354.17 \mathrm{~s}$ \\
\cline { 2 - 8 } & Iterations & 17 & 19 & 19 & 18 & 21 \\
\hline
\end{tabular}

From the table 1, it can be seen that the performance of GMRES(30) is superior to other iterative methods. On the other hand, we can find that the iteration number increases slowly with the number of DOFs, and the iteration number is fairly small relative to the number of unknowns.

\section{Conclusions}

Base on the results of numerical experiments we conclude that preconditioned krylov subspace methods are effective solvers for the solution of large scale dense nonsymmetric linear systems. The preconditioning is indispensable to improving the iterative efficiency of large scale problems. There is a trade-off between the cost of the constructing and applying the preconditioner, and the gain of convergence speed.

Acknowledgments. This work is supported by the Natural Science Foundation of China (No. 50475081).

\section{References}

1. H. Wang, Z. Yao, P. Wang.: On the preconditioners for fast multipole boundary element methods for 2D multi-domain elastostatics. Engng. Anal. Bound. Elem. 29 (2005) 673-688

2. Leung CY, Walker SP.: Iterative solution of large three-dimensional BEM analyses using GMRES technique. Int. J. Numer. Methods Eng. 40 (1997) 2227-36

3. Valente FP, Pina HLG.: Conjugate gradient methods for three-dimensional BEM systems of equations. Engng. Anal. Bound. Elem. 30 (2006) 441-449

4. S. Amini, N. D. Maines.: Preconditioned Krylov subspace methods for boundary element solution of the Helmholtz equation. Int. J. Numer. Methods Eng. 41 (1998) 875-898 